\title{
Energy Levels of the Coaxial Internal Rotator with Rectangular Potential Function
}

\author{
J. O. HALFord and G. L. Vidale* \\ Department of Chemistry, University of Michigan, Ann Arbor, Michigan
}

(Received February 20, 1956)

\begin{abstract}
For the coaxial internal rotator with rectangular potential function and freely variable valley breadth at constant periodicity, conditions are specified for an infinite number of points at which the borders of consecutive bands of energy levels must be exactly degenerate. As the valley breadth is changed through a degeneracy, the level of one symmetry relative to the potential function moves from the upper to the lower band of energy levels, or vice versa.

It follows that in the rotational region above the potential maximum the levels at the band limits can repeatedly cross and re-cross when the potential maximum is increased with all other factors constant.

Similar behavior will presumably occur with other potential functions.
\end{abstract}

$I^{N}$ $\mathrm{N}$ the calculation of the energies at the band limits for an internal rotator with the potential function

$$
2 V=V_{0}(1-\cos 3 x)+V_{0}(1-\cos 6 x) / a
$$

an irregularity in the order of the levels was obtained for $a=+4$ as compared with the simple sinusoidal case (infinite $a$ ) or the cases with finite negative $a$. All of the energies at the band limits can be obtained with two assignments of a coupling or phase constant, giving rise to two forms of the secular equation each of which can be split into two factors. One of the factors, made up of elements which are formed by addition from the elements of corresponding rows and columns of the original secular determinant, can be called even, while the other, which involves the corresponding substractions, is designated as odd.

The bands can also be classed even and odd by assigning index numbers $(l)$ beginning with zero for the lowest band. In all trial calculations with infinite or negative $a$, the levels at the borders of the even bands came from the even determinants and those in the odd bands came from the odd factors. With $a=+4$, at the border between the third and fourth band, however, the even determinant provided a level in the odd band and vice versa. This indicates that the symmetry of the wave function at a band limit depends not only upon the order number of the band but also upon the value of the constant $a$ in Eq. (1).

Examination for symmetry of the wave functions of the family of rotators represented by Eq. (1) presents too detailed a problem for convenient solution. If, however, a rectangular potential function of like symmetry and periodicity is substituted for Eq. (1), the waves are readily obtained and conclusions about their symmetry should be qualitatively valid for the models with trigonometric potentials. The sum of barrier and valley widths, $Q$ and $P$, respectively, is held constant at the appropriate fraction of $2 \pi$, and the ratio $Q / P$ is varied to produce an effect similar to that of changing $a$ in Eq. (1.) In the first approximation it can be taken

\footnotetext{
* Present address, Department of Chemistry, University of
} Southern California, Los Angeles, California. that two models are comparable which have the same average value of the potential energy over the cycle, $V_{0} Q /(P+Q)$ in the one system and $V_{0}(a+1) /(2 a)$ in the other. The analogy breaks down, however, for $-4<a<+4$, a range in which the potential function of Eq. (1) acquires extra maxima and minima.

Although the rectangular internal rotator is presumably widely understood, very little published information about the derivation of the secular equation and the wave functions is available. For the ethane-like model, however, Charlesby ${ }^{1}$ has presented a complete treatment which, with the substitution of the general symmetry number $n$ in the equations, is valid for all ratios $P / Q$ and all symmetry numbers. A general discussion of problems of this type has been given by Koenig. ${ }^{2}$

Application of the boundary conditions perhaps deserves further discussion here. This involves the use of a theorem of Bloch, ${ }^{3}$ which is in effect a somewhat generalized form of the Floquet ${ }^{4}$ theorem. This theorem states that the Schroedinger equation with a periodic potential function of period $l$ has solutions of the form,

$$
\psi(x)=e^{\mu x} X(x)
$$

where $X(x)$ is also a periodic function of period $l$ and $\mu$ is a constant for each of several sets of solutions. It is clear from the proof of the theorem that Eq. (2) would be valid if $X(x)$ had a period which was an integral multiple of the period of the potential function, but that $\mu$ would now be different.

It is therefore permissible to write

$$
e^{-i \delta x} \psi(x)=e^{i(\sigma-\delta) x} P(x)=X(x)
$$

in which the exponential factors are chosen imaginary to yield finite functions, $X(x)$ has the period of the potential function, and $P(x)$ has the period $2 \pi$. From

\footnotetext{
1 A. Charlesby, Proc. Phys. Soc. (London) 54, 471 (1942).

2 H. D. Koenig, Phys. Rev. 44, 657 (1933).

${ }^{3}$ F. Bloch, Z. Physik 52, 555 (1928).

${ }^{4} \mathrm{H}$. Margenau and G. M. Murphy, The Mathematics of Physics and Chemistry (D. Van Nostrand Company, Inc., New York, 1943), p. 80.
} 
Eq. (3), it readily follows that, since

$$
\begin{gathered}
P(0)=P(2 \pi)=X(0)=X(2 \pi), \\
\sigma=\delta+s,
\end{gathered}
$$

where $s$ is any integer.

The evaluation of $\sigma$ presented by Koehler and Dennison $^{6}$ for the rotator with sinusoidal potential function is valid for the rectangular potential function as well as any other for which $\psi(x)=e^{i \sigma x} P(x)$ as in Eq. (3). They write the wave function for the entire motion about the axis of the internal rotation as

with

$$
\psi(x, \varphi)=e^{i K \varphi} e^{i \sigma x} P(x)
$$

and

$$
\varphi=C_{1} \varphi_{1} / C+C_{2} \varphi_{2} / C
$$

$$
x=\varphi_{1}-\varphi_{2} .
$$

Here $\varphi_{1}$ and $\varphi_{2}$ describe the rotational positions of the individual opposed coaxial rotators, $C_{1}$ and $C_{2}$ are the corresponding individual moments of inertia, and $C=C_{1}+C_{2}$. It is required that $\psi(x, \varphi)$ be invariant when either $\varphi_{1}$ or $\varphi_{2}$ is changed by a multiple of $2 \pi$.

Introduction of this requirement into Eqs. (5) and (6) leads to the conclusion that $K$ is any integer and

$$
\sigma=\mp K C_{1} / C+s_{1}, \quad \text { or } \pm K C_{2} / C+s_{2},
$$

where $s_{1}$ and $s_{2}$ are integers such that a general solution is obtained if either one is arbitrarily assigned. Koehler and Dennison have chosen $s_{1}=0$ and $\sigma=-K C_{1} / C$.

The wave function in any periodic interval $2 \pi / n$ of the potential function will consist of two smoothly connected segments of sine waves of two different wavelengths. In the valley intervals the wavelength is determined by the total energy measured from the bottom of the valley, in the barrier intervals by the excess of energy above the potential maximum. The general solution is written separately for the barrier and valley intervals, giving rise to four integration constants in the periodic interval from zero to $2 \pi / n$. If, for the function $X(x)$ of $\mathrm{Eq}$. (3), the ordinates and slopes are adjusted at $x=0, x=P$ and $x=2 \pi / n$ to produce continuity and a period of $2 \pi / n$, elementary systematic elimination of the integration constants will yield the secular equation.

If

$$
\beta^{2}=8 \pi^{2} I w / h^{2} \text { and } \gamma^{2}=8 \pi^{2} I(w-v) / h^{2},
$$

where $w$ and $v$ are the total energy and potential energy in ergs/molecule and $I$ is the reduced moment of inertia $C_{1} C_{2} / C$, the secular equation, with $\delta$ expressed in terms of the known constant $\sigma$ according to Eq. (4), takes the form

$$
\begin{array}{r}
\cos \beta P \cos \gamma Q-\left[\left(\beta^{2}+\gamma^{2}\right) / 2 \beta \gamma\right] \sin \beta P \sin \gamma Q \\
=\cos [2 \pi(\sigma+s) / n]
\end{array}
$$

${ }^{5}$ J. S. Koehler and D. M. Dennison, Phys. Rev. 57, 1006 (1940). or

$$
\begin{array}{r}
\cos \theta_{1} \cos \theta_{2}-\left[\left(\theta_{1}{ }^{2} Q / P+\theta_{2}{ }^{2} P / Q\right) / 2 \theta_{1} \theta_{2}\right] \sin \theta_{1} \sin \theta_{2} \\
=\cos [2 \pi(\sigma+s) / n]
\end{array}
$$

where

$$
\theta_{1}=2 P(\alpha E / V)^{\frac{1}{2}} \text { and } \theta_{2}=2 Q(\alpha[E / V-1])^{3}
$$

with $\alpha$ defined, after Koehler and Dennison, as

$$
\alpha=2 \pi^{2} I v / h^{2} .
$$

The energies, total and potential, are now expressed as $E$ and $V$, which may be in any units since only the ratio $E / V$ appears in Eq. (10).

Selection of a particular $\alpha, n$, and $\sigma / K$ will define the model and lead to one specific complete set of energy level ratios $E / V$. All permissible values of the function will be obtained with any $n$ consecutive integers $s$. Any other integer will merely reproduce the results obtained with one of the chosen set of $n$. Thus, for the common case with symmetry number three, there are three sets of answers, corresponding to the integers $-1,0$, and +1 for $s$.

Although $\gamma$ and $\theta_{2}$ are imaginary when $E<V$, real solutions for energy levels are derivable with the appropriate substitution of hyperbolic functions in the energy range from zero to $V$.

When $F(E / V)$, the left side of Eq. (10), is plotted against $E / V$, or better, $(E / V)^{\frac{1}{2}}$ a type of damped oscillatory curve is obtained. The term on the right defines a set of horizontal lines all lying in the range $-1<F(E / V)<+1$, such that every intersection with the plotted function represents an energy level of the model. The function, however, repeatedly leaves and re-enters the region of these lines. Each time the function crosses the region of permissible energies, a band of energy levels is outlined. When the function leaves this region and re-enters at higher $E / V$, a forbidden space between bands is encountered. This type of function has been plotted by Charlesby and by Pitzer and Gwinn ${ }^{6}$ and will not be reproduced here.

It is evident that the function will never have a maximum or minimum in the range $-1<F(E / V)<+1$ and that it cannot cross itself. Consequently there can be no overlapping of the bands of energy levels. The interval between two energy levels representing the limits of two consecutive bands will always be a forbidden region. The original irregularity found with rotators following Eq. (1) therefore suggests that a change of $a$ may move the symmetrical wave from the even to the odd band, or vice versa. A parallel effect can be expected with the rotators of Eq. (10) when the ratio $Q / P$ is changed. It seems likely that between two ratios $Q / P$ for which such a reversal has occurred, there will be a value of $Q / P$ at which the symmetrical and antisymmetrical waves are degenerate, where the forbidden

${ }^{6}$ K. S. Pitzer and W. D. Gwinn, J. Chem. Phys. 10, 428 (1942). 
TABLE I. Degeneracies between bands 3 and 4 for a rotator with rectangular potential.

\begin{tabular}{cccccc}
\hline \hline \multicolumn{7}{c}{$n=3$} & and & $\alpha=4$ \\
\hline$R$ & 36 & 36.267 & 36.429 & 36.408 & 36 \\
$3 P / 2 \pi$ & 0 & 0.2147 & 0.4457 & 0.7011 & 1 \\
$E / V$ & 3.25 & 3.0520 & 2.8311 & 2.5744 & $(2.25)$ \\
$m_{1}$ & 0 & 1 & 2 & 3 & 4 \\
$m_{2}$ & 4 & 3 & 2 & 1 & 0 \\
\hline
\end{tabular}

interval between the corresponding two bands has disappeared.

It is readily demonstrated that such degeneracies must occur. If $\theta_{1}=m_{1} \pi$ and $\theta_{2}=m_{2} \pi$, where $m_{1}$ and $m_{2}$ are integers, $F(E / V)$ will have the value \pm 1 , which is the condition for the band limits. From the definition of $\theta_{1}$ and $\theta_{2}$, with free choice of $Q / P$, it is evident that a real energy and a fixed $Q / P$ are determined for any rotator with a characteristic $\alpha$ when the integers $m_{1}$ and $m_{2}$ are chosen. In addition, for this condition, every term in the derivative of $F(E / V)$ with respect to $E / V$ will contain either $\sin \theta_{1}$ or $\sin \theta_{2}$ as a factor. The function is therefore tangent at a maximum or minimum to the limiting line at $F(E / V)= \pm 1$. This tangency is obviously the condition for degeneracy.

For a family of rotators with a fixed barrier height and reduced moment of inertia and a freely variable $P$ and $Q$, an infinite number of these degeneracies will occur. Each one, distinguished by a particular choice of the multipliers $m_{1}$ and $m_{2}$, can be located relative to the assigned order numbers of the bands in the following manner:

Let $R$ be a measure of the energy in excess of the average potential energy, as in Eq. (12).

$$
R=4 \alpha(E / V-Q n / 2 \pi) \text {. }
$$

Since $\theta_{1}+\theta_{2}=\left(m_{1}+m_{2}\right) \pi$, it follows, for $Q$ small enough to permit substitution of $(E / V-1)$ by $E / V$ without serious loss of accuracy, that as $Q$ approaches zero,

and

$$
2(\alpha E / V)^{\frac{1}{2}}(P+Q) \rightarrow\left(m_{1}+m_{2}\right) \pi
$$

$$
R \rightarrow n^{2}\left(m_{1}+m_{2}\right)^{2} / 4-2 \alpha Q n / \pi .
$$

When $P$ is small, $E / V$ is substituted by $(E / V-1)$ to give

$$
R \rightarrow n^{2}\left(m_{1}+m_{2}\right)^{2} / 4+2 \alpha P n / \pi .
$$

At intermediate values of $P$ and $Q, R$ will tend toward an average of the limiting values of Eqs. (14) and (15) and will therefore remain in the region near $n^{2}\left(m_{1}+m_{2}\right)^{2} / 4$.

The introduction of a low potential barrier into a free internal rotator causes the separation of the energy levels into bands at the points where $R$ is the square of an integral multiple of $n / 2$, that is, at $R=n^{2} l^{2} / 4$, where $l$ is the index number of the higher band at the point of separation. Therefore, for $\theta_{1}=m_{1} \pi, \theta_{2}=m_{2} \pi$ and $m_{1}+m_{2}=l$, bands $l$ and $l-1$ will be contiguous.

The number of $Q / P$ values which will produce de- generacy at this particular border is given by the number of combinations of positive integers, $m_{1}$ and $m_{2}$ which have the same total $l$. If the trivial limiting free rotators for which $P=0$ or $Q=0$ are included, there are $l+1$ degeneracies, otherwise the number is $l-1$. For example, at the border between the third and fourth bands, $m_{1}+m_{2}=4$ and there are three $Q / P$ ratios for which neither $P$ nor $Q$ is zero at which the forbidden interval between the bands can be made to disappear.

This case is illustrated in Table I for a rotator with $\alpha=4$ and $n=3$. The table includes the trivial limiting free rotators for which $P$ is zero or $2 \pi / 3$. The $E / V$ ratio shown in the last column has no meaning in the limit where the potential barrier has completely disappeared. It is significant, however, for extremely narrow barrier regions of height $V$.

With degeneracies at three finite $Q / P$ ratios, the range of $Q / P$ values is divided into four intervals. In the first and third intervals, starting at one end of the $P$ or $Q$ scale, the borderline state of higher energy has a wave function which is symmetrical about the symmetry points of the potential energy function at the centers of the valley and barrier regions. In the second and fourth regions the wave function of higher energy is antisymmetrical in relation to these points. This has been checked by determining the wave forms for several pairs of levels near but on opposite sides of a degeneracy point. In each trial case the wave of one symmetry has always passed from one band to the other at the degeneracy. This behavior is more readily checked by examining the direction of the initial perturbation produced at the degeneracy point by a slight increase of the breadth of the barrier region. In the limit only one level is perturbed, always toward higher energy when $Q$ is increased, but the two perturbed waves at consecutive degeneracy points in the same border region are always in opposite symmetry classes.

Except for the symmetry specifications of the waves, the same effects will occur in all borderline regions. Where $R$ is near the square of an even multiple of $n / 2$, as in the above illustration, the waves are symmetrical or antisymmetrical in relation to both valley and barrier centers. The corresponding energies are given by Eqs. (9) and (10) when $F(E / V)=+1$, that is, with $\sigma$ and $s$ both zero. In the remaining half of the borderline regions, with $R$ near the square of an odd multiple of $n / 2$, the wave is symmetrical about the valley center and zero at the barrier center, or vice versa. Here the corresponding energies are obtained with $F(E / V)=-1$, that is, with $s=0$ and $\sigma=n / 2$.

When $P$ and $Q$ are equal, the rectangular model can be taken to be the analog of the rotator of Eq. (1) with infinite $a$, that is, with a sinusoidal potential function. In this case, for $n=3, \alpha=4, m_{1}+m_{2}=4$, both by direct determination of the wave form and by consideration of the perturbation effect, the antisymmetrical wave of the rectangular rotator occurs in the upper, or even- 
numbered band. This should also be true for the sinusoidal rotator. With $m_{1}+m_{2}=2$, however, the symmetrical wave is in the even-numbered band. It therefore appears that, in spite of the original observation for the sinusoidal case that the roots of the "even" determinant represented levels in the even-numbered bands, two consecutive levels obtained from the same determinant are actually in opposite symmetry classes.

The foregoing discussion has been limited to the effects produced by changing $P$ and $Q$ while the potential maximum is held constant. It is also of interest to examine the effect of changing the potential maximum at constant $Q / P$. The discussion is general if it is limited to a single degeneracy point characterized by the choice of an arbitrary pair of integers $m_{1}$ and $m_{2}$. From the definition equations, it follows that

$$
Q / P=\left(m_{2} / m_{1}\right)(E /[E-V])^{1} .
$$

It is clear that by proper choice of $\alpha$, that is, of $V$ at constant $I$, the particular band border can be made to appear, at will, anywhere on the $E / V$ scale. At very high energies $Q / P$ approaches $m_{2} / m_{1}$, while at $E=V$, it becomes infinite, and at $E<V$, imaginary. It is evident that, as $V$ is increased from low values, the $Q / P$ ratio required for a designated degeneracy must increase from $m_{2} / m_{1}$ to infinity as the corresponding band borderline is brought down to the level $E=V$. There can be no degeneracies between bands at energies equal to or less than $V$. The behavior under consideration can occur only in the rotational region above the barrier, and will be practically detectable only at relatively low energies in this region, since at high energy the band borders approach general degeneracy at all $Q / P$ ratios.

When, for a chosen constant $Q / P$, the barrier height is increased, every degeneracy for which the limiting low value of $Q / P$ at $m_{2} / m_{1}$ is less than the chosen $Q / P$ will cross this chosen point as $V$ is increased through the appropriate critical value. When this occurs, the symmetrical wave will pass from one band of levels into the other, reversing its position on the energy scale relative to the wave of opposite symmetry. The new orientation will obtain until a value of $V$ is reached where the degeneracy at $m_{2}-1$ and $m_{1}+1$ coincides with the chosen $Q / P$, when reversal to the original relative positions will occur.

It follows that in the original calculations with rotators following Eq. (1), limitation of the reversal to positive $a$ was fortuitous. The effect can be found with negative or infinite $a$ with proper choice of the model. It is more likely to be observed however, in the region of low positive $a$, corresponding to $Q \gg P$.

Koehler and Dennison and later, Pitzer and Gwinn, have stated that the levels of the internal rotator cannot cross as the barrier height is changed, presumably with all other factors constant, including the equivalent of the $Q / P$ ratio. Since they do not define "level" in connection with this statement, the assertion is not necessarily rendered invalid by present considerations. It is clear, however, that, if levels are characterized by the symmetry of the wave function relative to that of the potential function, those at the band borders may repeatedly cross and re-cross as the barrier is increased.

The wave functions for the rotator with a rectangular potential barrier are particularly simple at the degeneracy points in that every valley or barrier interval contains an integral number of half-waves. Since the number of half-waves in a periodic interval is always $m_{1}+m_{2}$ at the corresponding band border, the requirement of an integral number in each valley or barrier interval can be met exactly as often as the occurrence of the degeneracies. By inspection, such a wave can be fitted into the periodic interval with either required symmetry without changing the wavelength (energy) or periodicity. Consequently, waves of two symmetry classes must be degenerate under the stated condition.

If the valley extends from zero to $P$ on the $x$ scale, while the barrier lies between $P$ and $2 \pi / n$, the two waves, for the degeneracy condition, will have the form in the first interval from zero to $2 \pi / n$, without normalization,

$$
\left.\begin{array}{l}
y_{1}=\sin \beta x \\
y_{2}=\mp(\beta / \gamma) \sin \gamma(x-P)
\end{array}\right\} \text { and }\left\{\begin{array}{l}
y_{1}=\cos \beta x, \\
y_{2}=\mp \cos \gamma(x-P),
\end{array}\right.
$$

where $y_{1}$ applies to the valley region and $y_{2}$ to the barrier region. The minus sign is required for an odd number ( $m_{1}$ odd) of half-waves in the valley interval, the plus sign for an even number. The first wave function has zero amplitude at the potential discontinuities and requires the factor $\beta / \gamma$ to equate the slopes at these points. In the second case the slopes are zero at the discontinuities, with the result that the amplitude ratio does not require adjustment.

To write the wave functions in the higher periodic intervals for the degenerate pairs requires adjustment of the phase angle by the appropriate integral multiple of $2 \pi / n$. For the regions at the upper borders of the even numbered bands, the function will reverse its sign in successive periodic intervals.

It is unlikely, but not impossible, that the details of the internal rotator energy level system brought out here will find experimental utility. Transitions involving pairs of states which can become degenerate will occur with practically equal probability. The present discussion calls attention to a possible irregularity in the spacing of the corresponding spectral lines. It is also possible that a degeneracy might occur at or near the $Q / P$ ratio or its equivalent which characterizes a real molecule. If this happened in the lowenergy rotational region, one or more lines might be missing from the spectrum. 